\title{
Fixed point theorems for monotone operators and applications to nonlinear elliptic problems
}

Jun $\mathrm{Wu}^{1}$ and Yicheng Liu ${ }^{2 *}$

\section{"Correspondence:}

liuyc2001@hotmail.com

${ }^{2}$ Department of Mathematics and

Systems Science, College of

Science, National University of

Defense Technology, Changsha,

410073, P.R. China

Full list of author information is

available at the end of the article

\section{黛 Springer}

\begin{abstract}
In this paper, some fixed point theorems for monotone operators in partially ordered complete metric spaces are proved. Especially, a sufficient and necessary condition for the existence of a fixed point for a class of monotone operators is presented. The main results of this paper are generalizations of the recent results in the literature. Also, the main results can be applied to solve the nonlinear elliptic problems and the delayed hematopoiesis models.
\end{abstract}

MSC: $47 \mathrm{H} 10 ; 54 \mathrm{H} 25$

Keywords: coupled fixed point; partially ordered metric space; mixed monotone mapping; quadruple fixed point

\section{Introduction}

In the last decades, the fixed point theorems for the contraction mappings have been improved and generalized in different directions. During the extensive applications to the nonlinear integral equations, there were many researchers to investigate the existence of a fixed point for contraction-type mappings in partially ordered metric spaces. In 2006, Bhaskar and Lakshmikantham [1] introduced the notion of coupled fixed point and proved some coupled fixed point theorems for mixed monotone mappings. Later, Lakshmikantham and Ciric presented a coincidence point theorem for a mapping with $g$-monotone property in [2]. Also, the concepts of tripled fixed point and quadruple fixed point were introduced by the authors in [3] and [4], respectively. Meanwhile, they proved the corresponding fixed point theorems. More details on the direction of the coupled fixed point theory and its applications can be found in the literature (see, e.g., [5-27]).

In this manuscript, we give a common method to deal with the existence of a coupled fixed point and the coincidence point for a class of mixed monotone mappings in a partially ordered complete metric space. Indeed, we establish some fixed point theorems for the monotone operators in the partially ordered complete metric space. Especially, we present the sufficient and necessary condition for the existence of a fixed point for a class of monotone operators. Our results improve and generalize the main results in the literature $[1-4,10]$.

In the rest of this section, we recall some basic definitions.

Let $(X, \leq)$ be a partially ordered set, a subset $E \subset X$ is said to be a totally ordered subset if either $x \leq y$ or $y \leq x$ holds for all $x, y \in E$. We say the elements $x$ and $y$ are compa- 
rable if either $x \leq y$ or $y \leq x$ holds. It is said that a triple $(X, \leq, d)$ is a partially ordered complete metric space if $(X, \leq)$ is a partially ordered set and $(X, d)$ is a complete metric space. Let $\Phi$ denote all the functions $\phi:[0,+\infty) \rightarrow[0,+\infty)$ which satisfy that $\phi(r)<r$ and $\lim _{t \rightarrow r+} \phi(t)<r$ for all $r>0$. We should mention that Agarwal et al. [28] considered the non-decreasing functions $\phi:[0,+\infty) \rightarrow[0,+\infty)$ satisfying $\lim _{n \rightarrow \infty} \phi^{n}(r)=0$ for all $r>0$ and established some fixed point theorems.

Definition 1.1 (Bhaskar and Lakshmikantham [1]) Let $(X, \leq)$ be a partially ordered set and $F: X^{2} \rightarrow X$. The mapping $F$ is said to have the mixed monotone property if $F$ is monotone non-decreasing in its first argument and is monotone non-increasing in its second argument, that is, for any $x, y \in X$,

$$
\begin{array}{llll}
x_{1}, x_{2} \in X, & x_{1} \leq x_{2} \quad \Rightarrow \quad F\left(x_{1}, y\right) \leq F\left(x_{2}, y\right) \quad \text { and } \\
y_{1}, y_{2} \in X, & y_{1} \leq y_{2} \quad \Rightarrow \quad F\left(x, y_{2}\right) \leq F\left(x, y_{1}\right) .
\end{array}
$$

Definition 1.2 (Bhaskar and Lakshmikantham [1]) An element $(x, y) \in X^{2}$ is said to be a coupled fixed point of the mapping $F: X^{2} \rightarrow X$ if $F(x, y)=x$ and $F(y, x)=y$.

\section{Fixed points theorems for monotone operators}

Theorem 2.1 Let $(X, \preceq, \rho)$ be a partially ordered complete metric space and let $G: X \rightarrow X$ be a monotone non-decreasing operator with respect to the order $\preceq$ on $X$. Assume that

(i) there is a $\varphi \in \Phi$ such that

$$
\rho(G(\tilde{x}), G(\tilde{y})) \leq \varphi(\rho(\tilde{x}, \tilde{y})) \quad \text { for each } \tilde{x}, \tilde{y} \in X \text { with } \tilde{x} \preceq \tilde{y}
$$

(ii) there exists an $\tilde{x}_{0} \in X$ such that $\tilde{x}_{0} \preceq G\left(\tilde{x}_{0}\right)$;

(iii) either (a) $G$ is a continuous operator, or (b) if a non-decreasing monotone sequence $\tilde{x}_{n}$ in $X$ tends to $\bar{x}$, then $\tilde{x}_{n} \preceq \bar{x}$ for all $n$.

Then the operator $G$ has a fixed point in $X$.

Proof Definite a sequence $\left\{\tilde{x}_{n}\right\}$ in $X^{2}$ by

$$
\tilde{x}_{n}=G\left(\tilde{x}_{n-1}\right) \quad \text { for } n=1,2, \ldots
$$

Considering the operator $\tilde{F}$ is non-decreasing monotone for the order $\preceq$ and $\tilde{x}_{0} \preceq G\left(\tilde{x}_{0}\right)$, we have

$$
\tilde{x}_{0} \preceq \tilde{x}_{1} \preceq \tilde{x}_{2} \preceq \cdots \preceq \tilde{x}_{n} \preceq \cdots
$$

If there exists $n_{0}$ such that $\tilde{x}_{n_{0}}=\tilde{x}_{n_{0}+1}$, then $\tilde{x}_{n_{0}}=G\left(\tilde{x}_{n_{0}}\right)$ and $\tilde{x}_{n_{0}}$ is a fixed point of $G$. Then the result of Theorem 2.1 trivially holds.

Suppose now that $\tilde{x}_{n} \neq \tilde{x}_{n+1}$ for all $n$. Let $a_{n}=\rho\left(\tilde{x}_{n+1}, \tilde{x}_{n}\right)$, noting that the sequence $\left\{\tilde{x}_{n}\right\}$ is a non-decreasing sequence in $X^{2}$, we conclude that

$$
\begin{aligned}
a_{n+1} & =\rho\left(\tilde{x}_{n+2}, \tilde{x}_{n+1}\right)=\rho\left(G\left(\tilde{x}_{n+1}\right), G\left(\tilde{x}_{n}\right)\right) \\
& \leq \varphi\left(\rho\left(\tilde{x}_{n+1}, \tilde{x}_{n}\right)\right)=\varphi\left(a_{n}\right)<a_{n} \text { for } n=0,1, \ldots
\end{aligned}
$$


Thus we obtain that

$$
0<a_{n+2} \leq \varphi\left(a_{n+1}\right)<a_{n+1} \leq \varphi\left(a_{n}\right)<a_{n} .
$$

This implies that both sequences $\left\{a_{n}\right\}$ and $\left\{\varphi\left(a_{n}\right)\right\}$ are convergent. Set $\lim _{n \rightarrow \infty} a_{n}=a$. If $a>0$, noting $\lim _{t \rightarrow r+} \varphi(t)<r$ for all $r>0$, we have

$$
a=\lim _{n \rightarrow \infty} a_{n+1} \leq \lim _{n \rightarrow \infty} \varphi\left(a_{n}\right)=\lim _{r \rightarrow a+} \varphi(r)<a .
$$

This is a contradiction. Thus $\lim _{n \rightarrow \infty} \rho\left(\tilde{x}_{n+1}, \tilde{x}_{n}\right)=\lim _{n \rightarrow \infty} a_{n}=0$.

Now, we shall prove that $\left\{\tilde{x}_{n}\right\}$ is a Cauchy sequence in $X^{2}$. In fact, by $\varphi \in \Phi$, we can choose a positive sequence $\left\{\varepsilon_{m}\right\}$ with $\lim _{m \rightarrow \infty} \varepsilon_{m}=0$ and $\varepsilon_{m}^{*}=\sup _{0 \leq t \leq \varepsilon_{m}} \varphi(t)<\varepsilon_{m}$. For a fixed $m$, there exists a large enough positive number $N$ satisfying $a_{N} \leq \varepsilon_{m}-\varepsilon_{m}^{*}$. Let $\tilde{x} \in \Omega:=\left\{\tilde{x} \in X^{2}: \rho\left(\tilde{x}, \tilde{x}_{N}\right) \leq \varepsilon_{m}, \tilde{x}_{N} \preceq \tilde{x}\right\}$, then by the triangle inequality

$$
\begin{aligned}
\rho\left(G(\tilde{x}), \tilde{x}_{N}\right) & \leq \rho\left(G(\tilde{x}), G\left(\tilde{x}_{N}\right)\right)+\rho\left(G\left(\tilde{x}_{N}\right), \tilde{x}_{N}\right) \\
& \leq \varphi\left(\rho\left(\tilde{x}, \tilde{x}_{N}\right)\right)+a_{N}<\varepsilon_{m}^{*}+a_{N} \\
& \leq \varepsilon_{m}^{*}+\varepsilon_{m}-\varepsilon_{m}^{*}=\varepsilon_{m} .
\end{aligned}
$$

Also, $\tilde{x}_{N} \preceq G\left(\tilde{x}_{N}\right) \preceq G(\tilde{x})$. This means that the set $\Omega$ is invariant for the operator $G$. Clearly, $\tilde{x}_{N} \in \Omega$. Thus $\tilde{x}_{N+p} \in \Omega$ for all $p \in Z^{+}$. So, the sequence $\left\{\tilde{x}_{n}\right\}$ is a Cauchy sequence in $X^{2}$. Since $\left(X^{2}, \rho\right)$ is a complete metric space, there exists a point $\bar{x} \in X^{2}$ such that $\lim _{n \rightarrow \infty} \tilde{x}_{n}=\bar{x}$.

Suppose that $G$ is a continuous operator. Then, by definition of $\left\{\tilde{x}_{n}\right\}$, we have

$$
\bar{x}=\lim _{n \rightarrow \infty} \tilde{x}_{n}=\lim _{n \rightarrow \infty} G\left(\tilde{x}_{n-1}\right)=G(\bar{x}) .
$$

Let us assume that the assumption (b) holds, then $\tilde{x}_{n} \preceq \bar{x}$ for all $n \in Z^{+}$. Thus from the assumption (i), we have

$$
\begin{aligned}
\rho(\bar{x}, G(\bar{x})) & \leq \rho\left(\bar{x}, G\left(\tilde{x}_{n}\right)\right)+\rho\left(G\left(\tilde{x}_{n}\right), G(\bar{x})\right) \\
& \leq \rho\left(\bar{x}, \tilde{x}_{n+1}\right)+\varphi\left(\rho\left(\tilde{x}_{n}, \bar{x}\right)\right) \rightarrow 0 \quad \text { as } n \rightarrow \infty .
\end{aligned}
$$

So, $\rho(\bar{x}, G(\bar{x}))=0$. The proof of Theorem 2.1 is complete.

Let $F: X^{2} \rightarrow X$ be a mapping having the mixed monotone property on $X$ and define the operator $G: X^{2} \rightarrow X^{2}$ by

$$
G(\tilde{x})=(F(x, y), F(y, x)) \quad \text { for all } \tilde{x}=(x, y) \in X^{2} .
$$

It is easy to see that the coupled fixed points of $F$ is the fixed points of $G$ in $X^{2}$. Also, for $\tilde{t}=(x, y), \tilde{s}=(u, v) \in X^{2}$, we introduce a partial order $\preceq$ in $X^{2}$ given by

$$
\tilde{t} \preceq \tilde{s} \quad \Leftrightarrow \quad x \leq u \text { and } \quad v \leq y \text {. }
$$


Thus, if $F$ has the mixed monotone property on $X$, then the operator $G$ is non-decreasing monotone for the order $\preceq$. For $\tilde{t}=(x, y), \tilde{s}=(u, v) \in X^{2}$, let $\rho(\tilde{t}, \tilde{s}):=d(x, u)+d(y, v)$, then $\left(X^{2}, \rho\right)$ is a complete metric space provided $(X, d)$ is a complete metric space. Then, as a consequence of Theorem 2.1, we achieve the following corollary.

Corollary 2.1 Let $(X, \leq, d)$ be a partially ordered complete metric space and let $F: X^{2} \rightarrow X$ be a mapping having the mixed monotone property on $X$. Assume that

(i) there is a $\varphi \in \Phi$ such that $G: X^{2} \rightarrow X^{2}$ satisfying

$$
\rho(G(\tilde{x}), G(\tilde{y})) \leq \varphi(\rho(\tilde{x}, \tilde{y})) \text { for each } \tilde{x}, \tilde{y} \in X^{2} \text { with } \tilde{x} \preceq \tilde{y} ;
$$

(ii) there exists an $\tilde{x}_{0} \in X^{2}$ such that $\tilde{x}_{0} \preceq G\left(\tilde{x}_{0}\right)$;

(iii) one of (a) and (b) holds:

(a) $G$ is a continuous operator;

(b) if a non-decreasing monotone sequence $\tilde{x}_{n}$ in $X^{2}$ tends to $\bar{x}$, then $\tilde{x}_{n} \preceq \bar{x}$ for all $n$.

Then the operator $G$ has a fixed point in $X^{2}$, that is, there exist $x, y \in X$ such that

$$
x=F(x, y) \text { and } y=F(y, x) .
$$

Let $D=\left\{\tilde{x} \in X^{2}: \tilde{x}\right.$ and $G(\tilde{x})$ are comparable $\}$, then we have the following theorem.

Theorem 2.2 Let $(X, \leq, d)$ be a partially ordered complete metric space and let $F: X^{2} \rightarrow X$ be a mapping having the mixed monotone property on X. Assume that (i) in Theorem 2.1 and one of following conditions holds:

(a) $G$ is a continuous operator;

(b) if a monotone sequence $\tilde{x}_{n}$ in $X^{2}$ tends to $\bar{x}$, then $\tilde{x}_{n}$ and $\bar{x}$ are comparable for all $n$.

Then the operator $G$ has a fixed point in $X^{2}$ if and only if $D \neq \phi$. Furthermore, if $D$ is a totally ordered nonempty subset, then the operator $G$ has a unique fixed point in $X^{2}$.

Proof It is easy to see that all the fixed points of $G$ fall in the set $D$. Thus if the operator $G$ has a fixed point in $X^{2}$, then $D \neq \phi$.

We suppose $D \neq \phi$. If the condition (a) holds and $\tilde{x}_{0} \in D$, then there are two cases: $\tilde{x}_{0} \preceq$ $G\left(\tilde{x}_{0}\right)$ or $G\left(\tilde{x}_{0}\right) \preceq \tilde{x}_{0}$. For the first case, following Theorem 2.1, we claim that the operator $G$ has a fixed point in $X^{2}$. For the other case: $G\left(\tilde{x}_{0}\right) \preceq \tilde{x}_{0}$, noting the symmetry of the metric, we see that the formula (1) holds for $\tilde{y} \preceq \tilde{x}$. Thus

$$
\rho(G(\tilde{x}), G(\tilde{y})) \leq \varphi(\rho(\tilde{x}, \tilde{y})) \quad \text { for each } \tilde{x}, \tilde{y} \in X^{2} \text { satisfying } \tilde{x} \text { is comparable with } \tilde{y} \text {. }
$$

Constructing the same sequence $\left\{\tilde{x}_{n}\right\}$ in $X^{2}$ by $\tilde{x}_{n}=G\left(\tilde{x}_{n-1}\right)$, for $n=1,2, \ldots$, we have

$$
\cdots \preceq \tilde{x}_{n} \preceq \cdots \preceq \tilde{x}_{2} \preceq \tilde{x}_{1} \preceq \tilde{x}_{0} .
$$

For a mini-revise to the proof of Theorem 2.1 and resetting $\Omega:=\left\{\tilde{x} \in X^{2}: \rho\left(\tilde{x}, \tilde{x}_{N}\right) \leq \varepsilon_{m}, \tilde{x} \preceq\right.$ $\left.\tilde{x}_{N}\right\}$, we conclude that the sequence $\left\{\tilde{x}_{n}\right\}$ tends to a fixed point of $G$.

Now we assume the condition (b) holds. Similar to the case (a), we see that the monotone sequence $\left\{\tilde{x}_{n}\right\}$ is a Cauchy sequence and denote $\bar{x}$ as the limit point. Thus $\bar{x}$ is comparable 
with $\tilde{x}_{n}$ for all $n \in Z^{+}$. Then we have

$$
\begin{aligned}
\rho(\bar{x}, G(\bar{x})) & \leq \rho\left(\bar{x}, G\left(\tilde{x}_{n}\right)\right)+\rho\left(G\left(\tilde{x}_{n}\right), G(\bar{x})\right) \\
& \leq \rho\left(\bar{x}, \tilde{x}_{n+1}\right)+\varphi\left(\rho\left(\tilde{x}_{n}, \bar{x}\right)\right) \rightarrow 0 \quad \text { as } n \rightarrow \infty .
\end{aligned}
$$

Thus the operator $G$ has a fixed point $\bar{x}$ in $X^{2}$.

Next, we suppose that $D$ is a totally ordered nonempty subset. It is sufficient to prove the uniqueness of a fixed point of $G$. Let $\tilde{x}$ and $\tilde{y}$ be two fixed points of $G$, then $\tilde{x}$ is comparable with $\tilde{y}, G(\tilde{x})=\tilde{x}$ and $G(\tilde{y})=\tilde{y}$. Following the assumption (i), we have

$$
\rho(\tilde{x}, \tilde{y})=\rho(G(\tilde{x}), G(\tilde{y})) \leq \varphi(\rho(\tilde{x}, \tilde{y}))<\rho(\tilde{x}, \tilde{y}) .
$$

Thus $\rho(\tilde{x}, \tilde{y})=0$, that is, $\tilde{x}=\tilde{y}$. The proof of Theorem 2.2 is complete.

Following Theorem 2.1, we have the next two corollaries.

Corollary 2.2 ([1], Theorem 2.1) Let $(X, \leq)$ be a partially ordered set and suppose there is a metric $d$ on $X$ such that $(X, d)$ is a complete metric space. Let $F: X^{2} \rightarrow X$ be a continuous mapping having the mixed monotone property on $X$. Assume that there exists a $k \in[0,1)$ with

$$
d(F(x, y), F(u, v)) \leq \frac{k}{2}[d(x, u)+d(y, v)] \text { for each } x \leq u \text { and } v \leq y .
$$

If there exist $\left(x_{0}, y_{0}\right) \in X$ such that $x_{0} \leq F\left(x_{0}, y_{0}\right)$ and $F\left(y_{0}, x_{0}\right) \leq y_{0}$, then there exist $(x, y) \in$ $X$ such that

$$
x=F(x, y) \text { and } y=F(y, x) .
$$

Proof Taking $\varphi(r)=k r$ for $r \geq 0, \tilde{t}=(x, y), \tilde{s}=(u, v)$, if $\tilde{t} \preceq \tilde{s}$ then

$$
\begin{aligned}
\rho(G(\tilde{t}), G(\tilde{s})) & =d(F(x, y), F(u, v))+d(F(y, x), F(v, u)) \\
& \leq k[d(x, u)+d(y, v)]=\varphi(\rho(\tilde{s}, \tilde{t})) .
\end{aligned}
$$

Thus Corollary 2.1 is an immediate consequence of Theorem 2.1.

Corollary 2.3 ([1], Theorem 2.2) Let $(X, \leq)$ be a partially ordered set and suppose there is a metric $d$ on $X$ such that $(X, d)$ is a complete metric space. Assume that $X$ has the following property:

(i) if a non-decreasing sequence $x_{n} \rightarrow x$, then $x_{n} \leq x$ for all $n$;

(ii) if a non-increasing sequence $y_{n} \rightarrow y$, then $y \leq y_{n}$ for all $n$.

Let $F: X^{2} \rightarrow X$ be a mapping having the mixed monotone property on $X$. Assume that there exists a $k \in[0,1)$ with

$$
d(F(x, y), F(u, v)) \leq \frac{k}{2}[d(x, u)+d(y, v)] \text { for each } x \leq u \text { and } v \leq y .
$$


If there exist $\left(x_{0}, y_{0}\right) \in X$ such that $x_{0} \leq F\left(x_{0}, y_{0}\right)$ and $F\left(y_{0}, x_{0}\right) \leq y_{0}$, then there exist $(x, y) \in$ $X$ such that

$$
x=F(x, y) \text { and } y=F(y, x) .
$$

Proof It follows from Theorem 2.1 immediately.

Let $(X,\|\cdot\|)$ be a real Banach space and let $K$ be a cone. The relation $x \leq y$ holds if and only if $y-x \in K$. Denote $K^{+}=K \backslash\{\theta\}$ and $K_{f}=\left\{x \in K^{+}: \lambda f \leq x \leq \mu f\right.$ for some positive real numbers $\lambda$ and $\mu\}$ for a given $f \in K^{+}$. Let $M(x, y)=\inf \{\lambda: x \leq \lambda y\}$ and $d(x, y)=$ $\log (\max \{M(x, y), M(y, x)\})$ for $x, y \in K_{f}$. Then $d$ defines a metric on $K_{f}$ which is known as the Thompson metric [15]. More details about the Thompson metric can be found in the references [29-32]

At this stage, we state our main results in the real Banach space.

Theorem 2.3 Let $(X,\|\cdot\|)$ be a real Banach space, let $K \subset X$ a cone and $f \in K^{+}$. Suppose that $A_{i}: K_{f} \times K_{f} \rightarrow K_{f}$ are two mixed monotone maps satisfying $A_{i}\left(t x, t^{-1} y\right) \geq t^{i} A_{i}(x, y)$ and $A_{i}(f, f) \in K_{f}$ for $t \in(0,1), i=1, p$ with $0<p<1$. Let $A=A_{1}+A_{p}$ and assume that there exists a point $\left(x_{0}, y_{0}\right) \in K_{f} \times K_{f}$ such that

$$
x_{0} \leq A\left(x_{0}, y_{0}\right) \leq A\left(y_{0}, x_{0}\right) \leq y_{0} .
$$

Then $A$ has a unique fixed point in $K_{f}$, that is, there exists a unique point $x \in K_{f}$ such that $A(x, x)=x$.

In order to prove this result, we need some technique lemmas.

Lemma 2.1 ([10], Lemma 3.1) Under the assumptions of Theorem 2.3, there exists $\delta_{x, y} \in$ $(p, 1)$ such that

$$
A\left(t x, t^{-1} y\right) \geq t^{\delta_{x, y}} A(x, y) \quad \text { for all } t \in(0,1) \text { and } x, y \in K_{f},
$$

where

$$
\delta_{x, y}=\frac{\Delta_{x, y}+p}{\Delta_{x, y}+1} \quad \text { and } \quad \Delta_{x, y}=M\left(A_{1}(x, y), A_{p}(x, y)\right) .
$$

Lemma 2.2 Under the assumptions of Theorem 2.3, then

$$
d(A(x, y), A(u, v)) \leq \delta_{u, v} \max \{d(x, u), d(y, v)\} .
$$

Proof Noting that $e^{-d(x, y)} y \leq x \leq e^{d(x, y)} y$ for all $x, y \in K_{f}$, we have

$$
\begin{aligned}
A(x, y) & \geq A\left(e^{-d(x, u)} u, e^{d(y, v)} v\right) \\
& \geq A\left(e^{-\max \{d(x, u), d(y, v)\}} u, e^{\max \{d(x, u), d(y, v)\}} v\right) \\
& \geq e^{-\delta_{u, v} \max \{d(x, u), d(y, v)\}} A(u, v) .
\end{aligned}
$$


On the other hand, since $A\left(t x, t^{-1} y\right) \leq t^{\delta_{x, y}} A(x, y)$ for $t>1$, then we have

$$
\begin{aligned}
A(x, y) & \leq A\left(e^{d(x, u)} u, e^{-d(y, v)} v\right) \leq A\left(e^{\max \{d(x, u), d(y, v)\}} u, e^{-\max \{d(x, u), d(y, v)\}} v\right) \\
& \leq e^{\delta_{u, v} \max \{d(x, u), d(y, v)\}} A(u, v) .
\end{aligned}
$$

Thus $d(A(x, y), A(u, v)) \leq \delta_{u, v} \max \{d(x, u), d(y, v)\}$.

Lemma 2.3 Under the assumptions of Theorem 2.3, the successive sequences $\left\{x_{n}\right\}$ and $\left\{y_{n}\right\}$ are Cauchy sequences, where

$$
x_{n+1}=A\left(x_{n}, y_{n}\right), \quad y_{n+1}=A\left(y_{n}, x_{n}\right), \quad n=0,1, \ldots
$$

Proof Since $x_{0} \leq A\left(x_{0}, y_{0}\right) \leq A\left(y_{0}, x_{0}\right) \leq y_{0}$, it follows by an induction argument that

$$
x_{0} \leq x_{1} \leq \cdots \leq x_{n} \leq \cdots \leq y_{n} \leq \cdots \leq y_{1} \leq y_{0} .
$$

Noting that

$$
\begin{aligned}
A_{1}\left(y_{n}, x_{n}\right) & \leq A_{1}\left(y_{0}, x_{0}\right) \leq M\left(A_{1}\left(y_{0}, x_{0}\right), A_{p}\left(x_{0}, y_{0}\right)\right) A_{p}\left(x_{0}, y_{0}\right) \\
& \leq M\left(A_{1}\left(y_{0}, x_{0}\right), A_{p}\left(x_{0}, y_{0}\right)\right) A_{p}\left(y_{n}, x_{n}\right),
\end{aligned}
$$

we have, for all $n$,

$$
\Delta_{y_{n}, x_{n}}=M\left(A_{1}\left(y_{n}, x_{n}\right), A_{p}\left(y_{n}, x_{n}\right)\right) \leq M\left(A_{1}\left(y_{0}, x_{0}\right), A_{p}\left(x_{0}, y_{0}\right)\right):=\Delta_{0} .
$$

Thus

$$
\delta_{y_{n}, x_{n}}=\frac{\Delta_{y_{n}, x_{n}}+p}{\Delta_{y_{n}, x_{n}}+1} \leq \frac{\Delta_{0}+p}{\Delta_{0}+1}:=\delta_{0} .
$$

Next, we claim that

$$
x_{n} \geq e^{-d\left(x_{0}, y_{0}\right) \delta_{0}^{n}} y_{n}, \quad n=0,1,2, \ldots
$$

In fact, it holds for $n=0$. For arbitrary $n$, by induction argument, we have

$$
\begin{aligned}
x_{n+1}=A\left(x_{n}, y_{n}\right) & \geq A\left(e^{-d\left(x_{0}, y_{0}\right) \delta_{0}^{n}} y_{n}, e^{d\left(x_{0}, y_{0}\right) \delta_{0}^{n}} x_{n}\right) \\
& \geq\left(e^{-d\left(x_{0}, y_{0}\right) \delta_{0}^{n}}\right)^{\delta_{y_{n}, x_{n}}} A\left(y_{n}, x_{n}\right) \geq e^{-d\left(x_{0}, y_{0}\right) \delta_{0}^{n+1}} y_{n+1} .
\end{aligned}
$$

Thus (3) holds for all $n$.

On the other hand, since

$$
\begin{aligned}
A_{1}\left(x_{n}, y_{n}\right) & \leq A_{1}\left(y_{n}, x_{n}\right) \leq \Delta_{y_{n}, x_{n}} A_{p}\left(y_{n}, x_{n}\right) \\
& \leq \Delta_{0} A_{p}\left(y_{n}, x_{n}\right) \leq \Delta_{0} A_{p}\left(e^{d\left(x_{0}, y_{0}\right) \delta_{0}^{n}} x_{n}, e^{-d\left(x_{0}, y_{0}\right) \delta_{0}^{n}} y_{n}\right) \\
& \leq \Delta_{0} e^{p d\left(x_{0}, y_{0}\right) \delta_{0}^{n}} A_{p}\left(x_{n}, y_{n}\right) \leq \Delta_{0} e^{p d\left(x_{0}, y_{0}\right)} A_{p}\left(x_{n}, y_{n}\right),
\end{aligned}
$$


we obtain that, for all $n$,

$$
\Delta_{x_{n}, y_{n}}=M\left(A_{1}\left(x_{n}, y_{n}\right), A_{p}\left(x_{n}, y_{n}\right)\right) \leq \Delta_{0} e^{p d\left(x_{0}, y_{0}\right)}:=\Delta_{1} .
$$

Thus

$$
\delta_{x_{n}, y_{n}}=\frac{\Delta_{x_{n}, y_{n}}+p}{\Delta_{x_{n}, y_{n}}+1} \leq \frac{\Delta_{1}+p}{\Delta_{1}+1}:=\delta_{1} .
$$

Then following Lemma 2.2, we have

$$
\begin{aligned}
d\left(x_{n+1}, x_{n}\right) & =d\left(A\left(x_{n}, y_{n}\right), A\left(x_{n-1}, y_{n-1}\right)\right) \\
& \leq \delta_{x_{n-1}, y_{n-1}} \max \left\{d\left(x_{n}, x_{n-1}\right), d\left(y_{n}, y_{n-1}\right)\right\} \\
& \leq \delta_{1} \max \left\{d\left(x_{n}, x_{n-1}\right), d\left(y_{n}, y_{n-1}\right)\right\} .
\end{aligned}
$$

Similarly, we have

$$
\begin{aligned}
d\left(y_{n+1}, y_{n}\right) & =d\left(A\left(y_{n}, x_{n}\right), A\left(y_{n-1}, x_{n-1}\right)\right) \\
& \leq \delta_{y_{n-1}, x_{n-1}} \max \left\{d\left(y_{n}, y_{n-1}\right), d\left(x_{n}, x_{n-1}\right)\right\} \\
& \leq \delta_{0} \max \left\{d\left(x_{n}, x_{n-1}\right), d\left(y_{n}, y_{n-1}\right)\right\} .
\end{aligned}
$$

Let $\delta=\max \left\{\delta_{0}, \delta_{1}\right\}$, then $\delta<1$ and

$$
\max \left\{d\left(x_{n+1}, x_{n}\right), d\left(y_{n+1}, y_{n}\right)\right\} \leq \delta \max \left\{d\left(x_{n}, x_{n-1}\right), d\left(y_{n}, y_{n-1}\right)\right\}
$$

Thus, for all $n$,

$$
\max \left\{d\left(x_{n+1}, x_{n}\right), d\left(y_{n+1}, y_{n}\right)\right\} \leq \delta^{n} \max \left\{d\left(x_{1}, x_{0}\right), d\left(y_{1}, y_{0}\right)\right\}
$$

Furthermore, for any $k \in Z^{+}$, we have

$$
\max \left\{d\left(x_{n+k}, x_{n}\right), d\left(y_{n+k}, y_{n}\right)\right\} \leq \frac{\delta^{n}}{1-\delta} \max \left\{d\left(x_{1}, x_{0}\right), d\left(y_{1}, y_{0}\right)\right\}
$$

This shows that both successive sequences $\left\{x_{n}\right\}$ and $\left\{y_{n}\right\}$ are Cauchy sequences.

Proof of Theorem 2.3 By Lemma 2.3, there are $a, b \in K$ such that $\lim _{n \rightarrow \infty} x_{n}=a$ and $\lim _{n \rightarrow \infty} y_{n}=b$. Obviously, $x_{n} \leq a$ and $b \leq y_{n}$ for all $n \in Z^{+}$. Thus $a, b \in K_{f}$. Next, noting that

$$
d\left(x_{n+1}, A(a, b)\right)=d\left(A\left(x_{n}, y_{n}\right), A(a, b)\right) \leq \delta_{a, b} \max \left\{d\left(x_{n}, a\right), d\left(y_{n}, b\right)\right\}
$$

and

$$
d\left(y_{n+1}, A(b, a)\right)=d\left(A\left(y_{n}, x_{n}\right), A(b, a)\right) \leq \delta_{b, a} \max \left\{d\left(x_{n}, a\right), d\left(y_{n}, b\right)\right\}
$$


and letting $n$ go to infinity, we get that $a=A(a, b)$ and $b=A(b, a)$. It follows from

$$
d(a, b)=d(A(a, b), A(b, a)) \leq \delta_{b, a} \max \{d(b, a), d(a, b)\}=\delta_{b, a} d(a, b)
$$

that $d(a, b)=0$ and $a=b$. The uniqueness is obvious. Thus $A$ has a unique fixed point in $K_{f}$, that is, there exists a unique point $a \in K_{f}$ such that $A(a, a)=a$. The proof is complete.

Remark 2.1 Our result in Theorem 2.3 improved the corresponding result in [10] (Theorem 3.4) and removed some restriction conditions: the successive sequences have convergent subsequences.

\section{Application to the nonlinear elliptic problems}

Let $\Omega$ be the open unit ball in $R^{n}, n \geq 1$, with center at the origin. We consider positive solutions of the Dirichlet problem

$$
\left\{\begin{array}{l}
\nabla(a(|x|) \nabla(u))+b(|x|)\left(u^{p}+\frac{c}{10+u^{q}}\right)=0 \quad \text { in } \Omega, \\
u=0 \quad \text { on } \partial \Omega .
\end{array}\right.
$$

When $a(|x|)=b(|x|)=1, n \geq 3$ and $c=0$, it is well known that (4) has no positive solution if $p>\frac{n+2}{n-2}$, and that the positive solution of (4) is unique if $p>1$, see [22] and [23]. Also, in this case when $0<p<1,(4)$ has a unique positive radial solution [24].

In this section, we assume that $p \in(0,1], q \in(0,1)$ and $c>0$ are constants, $a(r)$ and $b(r)$ are positive and continuous for $0 \leq r \leq 1$. Our result is as follows.

Theorem 3.1 Problem (4) has a unique positive radial solution if $\frac{b_{\max }}{2 n a_{\min }}<1$, where $a_{\min }:=$ $\min \{a(r): r \in[0,1]\}$ and $b_{\max }:=\max \{b(r): r \in[0,1]\}$.

To this end, we should establish a technique lemma.

Lemma 3.1 The function $u$ is a positive radial solution of problem (4) if and only if $u$ is a positive solution of the integral equation

$$
u(r)=\int_{0}^{1} G(r, t) b(t)\left[u^{p}(t)+\frac{c}{10+u^{q}(t)}\right] d t,
$$

where

$$
G(r, t)= \begin{cases}\int_{t}^{1} \frac{1}{a(s)}\left(\frac{t}{s}\right)^{n-1} d s, & 0 \leq r<t \leq 1 \\ \int_{r}^{1} \frac{1}{a(s)}\left(\frac{t}{s}\right)^{n-1} d s, & 0 \leq t \leq r \leq 1\end{cases}
$$

Proof Assuming solutions to be functions of $r$, the radial distance from the origin, (4) reduces to

$$
\left\{\begin{array}{l}
\left(a(r) u^{\prime}(r)\right)^{\prime}+\frac{n-1}{r} a(r) u^{\prime}(r)+b(r)\left[u^{p}(r)+\frac{c}{10+u^{q}(r)}\right]=0, \quad 0<r<1, \\
u(1)=0,
\end{array}\right.
$$


where $u^{\prime}(r)=\frac{d}{d r} u(r)$. Then the Green function for problem (5) is

$$
G(r, t)= \begin{cases}\int_{t}^{1} \frac{1}{a(s)}\left(\frac{t}{s}\right)^{n-1} d s, & 0 \leq r<t \leq 1, \\ \int_{r}^{1} \frac{1}{a(s)}\left(\frac{t}{s}\right)^{n-1} d s, & 0 \leq t \leq r \leq 1,\end{cases}
$$

which is positive on $[0,1) \times[0,1)$. Thus the function $u$ is a positive radial solution of problem (4) if and only if $u$ is a positive solution of the integral equation

$$
u(r)=\int_{0}^{1} G(r, t) b(t)\left[u^{p}(t)+\frac{c}{10+u^{q}(t)}\right] d t .
$$

Proof of Theorem 3.1 Let $K$ denote the cone of nonnegative functions in $C[0,1]$, the relation $x \leq y$ holds if and only if $x(t) \leq y(t)$ for all $t \in[0,1], K^{+}=K \backslash\{0\}$ and $f(r)=1-r^{2}$ for $r \in[0,1]$, then $f \in K^{+}$and $0 \leq f(r) \leq f_{\max }=1$. Denote $K_{f}=\left\{x \in K^{+}: \lambda f \leq x \leq\right.$ $\mu f$ for some positive numbers $\lambda, \mu \in R\}$.

Now we introduce the maps $A_{1}, A_{q}: K_{f} \times K_{f} \rightarrow K_{f}$ defined by

$$
\begin{aligned}
& A_{1}(x, y)(r)=\int_{0}^{1} G(r, t) b(t) x^{p}(t) d t, \\
& A_{q}(x, y)(r)=\int_{0}^{1} G(r, t) b(t) \frac{c}{10+y^{q}(t)} d t .
\end{aligned}
$$

For $g, h \in K_{f}$, then there exist $\lambda_{g}, \lambda_{h}, \mu_{g}, \mu_{h}$ such that $\mu_{g} f \leq g \leq \lambda_{g} f$ and $\mu_{h} f \leq h \leq \lambda_{h} f$. By direct computation, we have

$$
\begin{aligned}
& \frac{b_{\min } \mu_{g}^{p}}{n(n+2) a_{\max }} f(r) \leq A_{1}(g, h)(r) \leq \int_{0}^{1} G(r, t) b(t) \lambda_{g}^{p} d t \leq \frac{b_{\max } \lambda_{g}^{p}}{2 n a_{\min }} f(r), \\
& \frac{c b_{\min }}{2 n a_{\max }\left(10+h_{\max }^{q}\right)} f(r) \leq A_{q}(g, h)(r) \leq \int_{0}^{1} G(r, t) b(t) \frac{c}{10} d t \leq \frac{c b_{\max }}{20 n a_{\min }} f(r) .
\end{aligned}
$$

Thus the map $A_{i}$ is well defined and $A_{i}(f, f) \in K_{f}$ for $i=1, q$. Also, $A_{1}(f, f)+A_{q}(f, f) \in K_{f}$. Obviously, $A_{i}$ is a mixed monotone map in $K_{f}$ and $A_{1}\left(t x, t^{-1} y\right) \geq t A_{1}(x, y)$ and $A_{q}\left(t x, t^{-1} y\right) \geq$ $t^{q} A_{q}(x, y)$.

Since $\frac{b_{\max }}{2 n a_{\min }}<1$, we choose a positive number $k$ (large enough) satisfying

$$
\frac{b_{\max }}{2 n a_{\min }}\left(k+\frac{c}{10}\right) \leq k \quad \text { and } \quad \frac{b_{\min }}{2 n a_{\max }} \frac{c}{10+k} \leq 1
$$

Let $A=A_{1}+A_{q}, x_{0}(r)=\frac{b_{\min }}{2 n a_{\max }} \frac{c}{10+k}\left(1-r^{2}\right), y_{0}(r)=k\left(1-r^{2}\right)$, then $x_{0}, y_{0} \in K_{f}$ and

$$
\begin{aligned}
A\left(x_{0}, y_{0}\right)(r) & =\int_{0}^{1} G(r, t) b(t)\left[x_{0}^{p}(t)+\frac{c}{10+y_{0}^{q}(t)}\right] d t \\
& \geq \frac{c b_{\min }}{10+k} \int_{0}^{1} G(r, t) d t \geq \frac{c b_{\min }}{2 n a_{\max }(10+k)}\left(1-r^{2}\right) \\
& =x_{0}(r)
\end{aligned}
$$


and

$$
\begin{aligned}
A\left(y_{0}, x_{0}\right)(r) & =\int_{0}^{1} G(r, t) b(t)\left[y_{0}^{p}(t)+\frac{c}{10+x_{0}^{q}(t)}\right] d t \\
& \leq \int_{0}^{1} G(r, t) b_{\max }\left[\frac{c}{10}+k f(t)\right] d t \\
& \leq\left(\frac{c}{10}+k\right) \frac{b_{\max }}{2 n a_{\min }} f(r) \\
& \leq k f(r)=y_{0}(r) .
\end{aligned}
$$

Thus

$$
x_{0} \leq A\left(x_{0}, y_{0}\right) \leq A\left(y_{0}, x_{0}\right) \leq y_{0} \text {. }
$$

Applying Theorem 2.3 to the operator $A$, we conclude that there is a unique point $u$ in $K_{f}$ such that $u(r)=A(u, u)(r)$. On the other hand, for all $g, h \in K^{+}$, we have

$$
\begin{aligned}
f(r)\left(g_{\max }^{p}+\frac{c}{10}\right) \frac{b_{\max }}{2 n a_{\min }} & \geq \int_{0}^{1} G(r, t) b(t)\left[g^{p}(t)+\frac{c}{10+h^{q}(t)}\right] d t \\
& \geq \frac{c b_{\min }}{2 n a_{\max }\left(10+h_{\max }^{q}\right)} f(r) .
\end{aligned}
$$

This means that $A(g, h) \in K_{f}$. Thus problem (4) has a unique positive radial solution.

\section{Application to the delayed hematopoiesis models}

In this section, we consider the positive periodic solution of the following hematopoiesis model with delays:

$$
x^{\prime}(t)=-a(t) x(t)+\sum_{i=1}^{n} \frac{b_{i}(t)}{1+x^{q}\left(t-\tau_{i}(t)\right)}
$$

where $a, b_{i}, \tau_{i} \in C(R, R)$ are positive $T$-periodic functions and $0 \leq \tau_{i}(t) \leq t$ for all $t \in[0, T]$, $q$ is a nonnegative constant $(i=1,2, \ldots, n)$. In the case when $q \geq 1, \mathrm{Wu}$ [26] proved that (6) had a unique positive $T$-periodic solution.

Here we assume that $q \in(0,1)$ and our result is as follows.

Theorem 4.1 Problem (6) has a unique positive T-periodic solution.

Proof Let $K$ denote the cone of nonnegative $T$-periodic functions in $C(R, R)$, the relation $x \leq y$ holds if and only if $x(t) \leq y(t)$ for all $t \in[0, T], K^{+}=K \backslash\{0\}$ and $f(r) \equiv 1$ for $r \in[0, T]$. Denote $K_{f}=\left\{x \in K^{+}: \lambda f \leq x \leq \mu f\right.$ for some positive numbers $\left.\lambda, \mu \in R\right\}$. It is easy to show that the function $x$ is a positive $T$-periodic solution of problem (6) if and only if $x$ is a positive solution of the integral equation

$$
x(r)=\frac{e^{\beta T}}{e^{\beta T}-1} \int_{r-T}^{r} e^{-\beta(r-s)}\left[(\beta-a(s)) x(s)+\sum_{i=1}^{n} \frac{b_{i}(s)}{1+x^{q}\left(s-\tau_{i}(s)\right)}\right] d s,
$$


where $\beta>a_{\max }$ is a constant. Define the maps $A_{1}, A_{q}: K_{f} \times K_{f} \rightarrow K_{f}$ by

$$
\begin{aligned}
& A_{1}(x, y)(t)=\frac{e^{\beta T}}{e^{\beta T}-1} \int_{t-T}^{t} e^{-\beta(t-s)}(\beta-a(s)) x(s) d s, \\
& A_{q}(x, y)(t)=\frac{e^{\beta T}}{e^{\beta T}-1} \int_{t-T}^{t} e^{-\beta(t-s)} \sum_{i=1}^{n} \frac{b_{i}(s)}{1+y^{q}\left(s-\tau_{i}(s)\right)} d s .
\end{aligned}
$$

For arbitrary $x, y \in K_{f}$, there are positive numbers $\lambda_{x}, \lambda_{y}, \mu_{x}, \mu_{y}$ such that $\lambda_{x} \leq x(t) \leq \mu_{x}$ and $\lambda_{y} \leq y(t) \leq \mu_{y}$ for all $t \in[0, T]$. Furthermore, we deduce that

$$
\begin{aligned}
A_{1}(x, y)(t) & =\frac{e^{\beta T}}{e^{\beta T}-1} \int_{t-T}^{t} e^{-\beta(t-s)}(\beta-a(s)) x(s) d s \\
& \geq \frac{e^{\beta T}}{e^{\beta T}-1} \int_{t-T}^{t} e^{-\beta(t-s)} d s\left(\beta-a_{\max }\right) \lambda_{x} \\
& =\left(1-\frac{a_{\max }}{\beta}\right) \lambda_{x}
\end{aligned}
$$

and

$$
A_{1}(x, y)(t) \leq\left(1-\frac{a_{\min }}{\beta}\right) \mu_{x}
$$

Similarly, we have

$$
\frac{1}{\beta} \sum_{i=1}^{n} \frac{b_{i \min }}{1+\mu_{y}^{q}} \leq A_{q}(x, y)(t) \leq \frac{1}{\beta} \sum_{i=1}^{n} \frac{b_{i \max }}{1+\lambda_{y}^{q}} \quad \text { for all } t \in[0, T] .
$$

This means $A_{1}(x, y) \in K_{f}$ and $A_{q}(x, y) \in K_{f}$. Thus $A_{1}$ and $A_{q}$ are well defined and $A_{1}(f, f), A_{q}(f, f) \in K_{f}$. Also, $A_{1}(f, f)+A_{q}(f, f) \in K_{f}$. Obviously, $A_{1}$ and $A_{q}$ are mixed monotone maps in $K_{f}$ and $A_{1}\left(t x, t^{-1} y\right) \geq t A_{1}(x, y)$ and $A_{q}\left(t x, t^{-1} y\right) \geq t^{q} A_{q}(x, y)$.

Since $\beta>a_{\max }$, we can choose a constant $k>1$ satisfying

$$
\left(1-\frac{a_{\min }}{\beta}\right) k+\frac{1}{\beta} \sum_{i=1}^{n} b_{i \max } \leq k \text { and } \quad \frac{1}{\beta} \sum_{i=1}^{n} \frac{b_{i \min }}{1+k^{q}}<1 .
$$

Let $A=A_{1}+A_{q}, x_{0}(t) \equiv \frac{1}{\beta} \sum_{i=1}^{n} \frac{b_{\text {imin }}}{1+k^{q}}$ and $y_{0}(t) \equiv k$ for all $t \in[0, T]$, then we have

$$
\begin{aligned}
A\left(x_{0}, y_{0}\right)(t) & =\frac{e^{\beta T}}{e^{\beta T}-1} \int_{t-T}^{t} e^{-\beta(t-s)}\left[(\beta-a(s)) x_{0}(s)+\sum_{i=1}^{n} \frac{b_{i}(s)}{1+y_{0}^{q}\left(s-\tau_{i}(s)\right)}\right] d s \\
& \geq \frac{e^{\beta T}}{e^{\beta T}-1} \int_{t-T}^{t} e^{-\beta(t-s)} \sum_{i=1}^{n} \frac{b_{i \min }}{1+k^{q}} d s \\
& =\frac{1}{\beta} \sum_{i=1}^{n} \frac{b_{i \min }}{1+k^{q}}=x_{0}(t)
\end{aligned}
$$


and

$$
\begin{aligned}
A\left(y_{0}, x_{0}\right)(t) & =\frac{e^{\beta T}}{e^{\beta T}-1} \int_{t-T}^{t} e^{-\beta(t-s)}\left[(\beta-a(s)) y_{0}(s)+\sum_{i=1}^{n} \frac{b_{i}(s)}{1+x_{0}^{q}\left(s-\tau_{i}(s)\right)}\right] d s \\
& \leq \frac{e^{\beta T}}{e^{\beta T}-1} \int_{t-T}^{t} e^{-\beta(t-s)}\left[\left(\beta-a_{\min }\right) k+\sum_{i=1}^{n} b_{i \max }\right] d s \\
& =\frac{1}{\beta}\left[\left(\beta-a_{\min }\right) k+\sum_{i=1}^{n} b_{i \max }\right] \leq k=y_{0}(t) .
\end{aligned}
$$

Thus

$$
x_{0} \leq A\left(x_{0}, y_{0}\right) \leq A\left(y_{0}, x_{0}\right) \leq y_{0} \text {. }
$$

Applying Theorem 2.3 to the operator $A$, we conclude that there is a unique point $x$ in $K_{f}$ such that $x(t)=A(x, x)(t)$. On the other hand, for all $g, h \in K^{+}$, we have

$$
f(t)\left[\frac{\sum_{i=1}^{n} b_{i \max }}{\beta}+\left(1-\frac{a_{\min }}{\beta}\right) g_{\max }\right] \geq A(g, h)(t) \geq \frac{1}{\beta} \sum_{i=1}^{n} \frac{b_{i \min }}{1+h_{\max }^{q}} f(t) .
$$

This means that $A(g, h) \in K_{f}$. Thus problem (6) has a unique positive $T$-periodic solution.

Remark 4.1 Using similar ideas, it is possible to extend our results to investigate the existence and uniqueness of nonlinear singular boundary value problems and fractional differential equation boundary value problems, which are mentioned extensively in the literature $[10,11,25]$.

\section{Competing interests}

The authors declare that they have no competing interests.

Authors' contributions

All authors contributed equally and read and approved the final manuscript.

\section{Author details}

${ }^{1}$ College of Mathematics and Computer Science, Changsha University of Science Technology, Changsha, 410114, P.R. China. ${ }^{2}$ Department of Mathematics and Systems Science, College of Science, National University of Defense Technology, Changsha, 410073, P.R. China.

\section{Acknowledgements}

The authors are grateful to the reviewers for their valuable comments and suggestions. This work was partly supported by the National Natural Science Foundation of China (11201481) and Hunan Provincial Education Department Foundation (12B007)

Received: 12 February 2013 Accepted: 8 May 2013 Published: 27 May 2013

\section{References}

1. Bhaskar, TG, Lakshmikantham, V: Fixed point theory in partially ordered metric spaces and applications. Nonlinear Anal. 65, 1379-1393 (2006)

2. Lakshmikantham, $\mathrm{V}$, Ciric, L: Couple fixed point theorems for nonlinear contractions in partially ordered metric spaces. Nonlinear Anal. 70, 4341-4349 (2009)

3. Berinde, V, Borcut, M: Tripled fixed point theorems for contractive type mappings in partially ordered metric spaces. Nonlinear Anal. 74(15), 4889-4897 (2011)

4. Karapinar, E, Luong, NV: Quadruple fixed point theorems for nonlinear contractions. Comput. Math. Appl. (2012). doi:10.1016/j.camwa.2012.02.061 
5. Abbas, M, Khan, MA, Radenovic, S: Common coupled fixed point theorems in cone metric space for $w$-compatible mappings. Appl. Math. Comput. 217, 195-203 (2010)

6. Amini-Harandi, A, Emami, H: A fixed point theorem for contraction type maps in partially ordered metric spaces and application to ordinary differential equations. Nonlinear Anal. 72, 2238-2242 (2010)

7. Chen, YZ: Existence theorems of coupled fixed points. J. Math. Anal. Appl. 154, 142-150 (1991)

8. Choudhury, BS, Kundu, A: A coupled coincidence point result in partially ordered metric spaces for compatible mappings. Nonlinear Anal. 73, 2524-2531 (2010)

9. Haghi, RH, Rezapour, S, Shahzad, N: Some fixed point generalizations are not real generalizations. Nonlinear Anal. 74, 1799-1803 (2011)

10. Huang, C-Y: Fixed point theorems for a class of positive mixed monotone operators. Math. Nachr. 285, 659-669 (2012)

11. Huang, MJ, Huang, CY, Tsai, TM: Hilbert's projective metric and nonlinear elliptic problems. Nonlinear Anal. 71, 2576-2584 (2009)

12. Figueroa, R, López Pouso, R: Coupled fixed points of multivalued operators and first-order ODEs with state-dependent deviating arguments. Nonlinear Anal. 74, 6876-6889 (2011)

13. Karapinar, E, Berinde, V: Quadruple fixed point theorems for nonlinear contractions in partially ordered metric spaces. Banach J. Math. Anal. 6, 74-79 (2012)

14. Karapinar, E: Couple fixed point theorems for nonlinear contractions in cone metric spaces. Comput. Math. Appl. 59, 3656-3668 (2010)

15. Thompson, AC: On certain contraction mappings in a partially ordered vector space. Proc. Am. Math. Soc. 14, 438-443 (1963)

16. Samet, B, Vetro, C, Vetro, P: Fixed point theorems for $\varphi$-contractive type mappings. Nonlinear Anal. 75, 2154-2165 (2012)

17. Shatanawi, W: Partially ordered cone metric spaces and coupled fixed point results. Comput. Math. Appl. 60, 2508-2515 (2010)

18. Shatanawi, W, Samet, B: On $(\psi, \phi)$-weakly contractive condition in partially ordered metric spaces. Comput. Math. Appl. 62, 3204-3214 (2011)

19. Shatanawi, W: On $\mathbf{w}$-compatible mappings and common coupled coincidence point in cone metric spaces. Appl. Math. Lett. 25, 925-931 (2012)

20. Xiao, JZ, Zhu, XH, Cao, YF: Common coupled fixed point results for probabilistic $\varphi$-contractions in Menger spaces. Nonlinear Anal. 74, 4589-4600 (2011)

21. Liu, YC, Li, ZX: Krasnoselskii type fixed point theorems and applications. Proc. Am. Math. Soc. 136, 1213-1220 (2008)

22. Gidas, B, Ni, W-M, Nirenberg, L: Symmetry and related properties via the maximum principle. Commun. Math. Phys. 68, 209-243 (1979)

23. Ni, W-M, Nussbaum, RD: Uniqueness and nonuniqueness for positive radial solutions of $\Delta u+f(u, r)=0$. Commun. Pure Appl. Math. 38, 67-108 (1985)

24. Bushell, PJ: The Cayley-Hilbert metric and positive operators. Linear Algebra Appl. 84, 271-280 (1986)

25. Zhai, C, Hao, M: Fixed point theorems for mixed monotone operators with perturbation and applications to fractional differential equation boundary value problems. Nonlinear Anal. 75, 2542-2551 (2012)

26. $\mathrm{Wu}, \mathrm{J}, \mathrm{Liu}, \mathrm{Y}$ : Fixed point theorems and uniqueness of the periodic solution for the hematopoiesis models. Abstr. Appl. Anal. 2012, Article ID 387193 (2012). doi:10.1155/2012/387193

27. Yang, XT: Existence and global attractivity of unique positive almost periodic solution for a model of hematopoiesis. Appl. Math. J. Chin. Univ. Ser. A 25, 25-34 (2010)

28. Agarwal, RP, El-Gebelly, MA, O’Regan, D: Generalized contractions in partially ordered metric spaces. Appl. Anal. 87, 109-116 (2008)

29. Guo, DJ, Lakshmikantham, V: Coupled fixed points of nonlinear operators with applications. Nonlinear Anal. 11, 623-632 (1987)

30. Guo, DJ: Fixed points of mixed monotone operators with applications. Appl. Anal. 31, $215-224$ (1988)

31. Guo, DJ: Existence and uniqueness of positive fixed points for mixed monotone operators and applications. Appl. Anal. 46, 91-100 (1992)

32. Guo, DJ, Cho, YJ, Zhu, J: Partial Ordering Methods in Nonlinear Problems. Nova Science Publishers, Hauppauge (2004)

doi:10.1186/1687-1812-2013-134

Cite this article as: Wu and Liu: Fixed point theorems for monotone operators and applications to nonlinear elliptic problems. Fixed Point Theory and Applications 2013 2013:134

\section{Submit your manuscript to a SpringerOpen ${ }^{\circ}$ journal and benefit from:}

- Convenient online submission

Rigorous peer review

- Immediate publication on acceptance

- Open access: articles freely available online

- High visibility within the field

- Retaining the copyright to your article 